\title{
THE NONUNIQUENESS OF IRREDUCIBLE CONSTITUENTS OF INTEGRAL GROUP REPRESENTATIONS ${ }^{1}$
}

\section{IRVING REINER}

Let $Z$ denote the ring of rational integers, $Q$ the rational field. As is well-known, the $Z$-representations of a finite group $G$ can be classified either according to $Q$-equivalence or according to $Z$-equivalence. Thus, two $Z$-representations $T, U$ of $G$ are $Q$-equivalent $\left(T \sim_{Q} U\right)$ if there exists a nonsingular rational matrix $P$ such that

$$
U(g)=P^{-1} T(g) P,
$$$$
g \in G \text {. }
$$

On the other hand, we write $T \sim_{z} U$ if (1) holds for some unimodular ${ }^{2}$ matrix $P$.

If a representation $T$ is equivalent to a "reduced" representation

$$
g \rightarrow\left(\begin{array}{cc}
T_{1}(g) & * \\
0 & T_{2}(g)
\end{array}\right), \quad g \in G,
$$

we say that $T$ is reducible. Conceivably we must distinguish between $Q$ - and $Z$-reducibility of a $Z$-representation. This difficulty does not in fact arise, because of the following theorem due to Zassenhaus [3].

(A) An integral representation is $Q$-reducible if and only if it is $Z$-reducible.

It is well-known that any given $Q$-representation $T$ of $G$ is $Q$ equivalent to a "completely reduced" representation

$$
\left(\begin{array}{ccc}
T_{1} & & 0 \\
& \ddots & \\
& & \cdot \\
0 & & T_{k}
\end{array}\right),
$$

in which the $T_{i}$ are irreducible. The Jordan-Hölder Theorem on modules asserts

(B) The irreducible representations $T_{1}, \cdots, T_{k}$ (often referred to as the irreducible constituents of $T$ ) are uniquely determined up to $Q$-equivalence and order of occurrence.

As an analogue of this, Zassenhaus [3] and Diederichsen [1] proved

(C) If the $Z$-representation $T$ is $Q$-equivalent to a completely re-

Presented to the Society, September 4, 1959; received by the editors September 22, 1959.

1 This work was supported in part by a contract with the Office of Naval Research.

2 A square matrix with integral entries and determinant \pm 1 is called unimodular. 
duced representation (2), then also $T$ is $Z$-equivalent to a reduced $Z$-representation

$$
\left(\begin{array}{lll}
U_{1} & & * \\
& \ddots & \\
0 & & U_{k}
\end{array}\right)
$$

in which

$$
U_{i} \sim{ }_{Q} T_{i}, \quad 1 \leqq i \leqq k .
$$

We may refer to $U_{1}, \cdots, U_{k}$ as a set of irreducible $Z$-constituents of $T$. From (B) it follows that they are unique up to $Q$-equivalence and order of occurrence. Diederichsen [1] (see also Maranda [2]) gave the following example to show that the irreducible $Z$-constituents were not necessarily unique up to $Z$-equivalence and order of occurrence.

Let

$$
G=\left\{a, b: a^{4}=b^{2}=(a b)^{2}=1\right\} .
$$

Then $G$ is the group of symmetries of the square, and $(G: 1)=8$. Set

(4) $A_{1}=\left(\begin{array}{rr}0 & 1 \\ -1 & 0\end{array}\right), \quad B_{1}=\left(\begin{array}{ll}0 & 1 \\ 1 & 0\end{array}\right) ; \quad A_{2}=\left(\begin{array}{rr}0 & 1 \\ -1 & 0\end{array}\right), B_{2}=\left(\begin{array}{rr}1 & 0 \\ 0 & -1\end{array}\right)$;

$$
T_{i}(a)=A_{i}, \quad T_{i}(b)=B_{i}, \quad i=1,2 .
$$

Then $T_{1}$ and $T_{2}$ are irreducible $Z$-representations of $G$ which are $Q$-equivalent but not $Z$-equivalent. However, for suitable choice of integral $\Lambda_{1}, \Lambda_{2}$, Diederichsen showed that

$$
\left(\begin{array}{ll}
T_{1} & \Lambda_{1} \\
0 & T_{1}
\end{array}\right) \sim_{Z}\left(\begin{array}{ll}
T_{2} & \Lambda_{2} \\
0 & T_{2}
\end{array}\right)
$$

Diederichsen attempted to show that this difficulty is due to the repetition of constituents, and asserted the following:

(D) Let $T$ be an integral representation of some finite group, and suppose that

$$
T \sim z\left(\begin{array}{lll}
U_{1} & & * \\
& \cdot & \\
& & \\
0 & & U_{k}
\end{array}\right)
$$

where for $i \neq j$, no irreducible constituent of $U_{i}$ is $Q$-equivalent to any irreducible constituent of $U_{j}$. Then $U_{1}, \cdots, U_{k}$ are unique up to $Z$-equivalence and order of occurrence. 
We shall show here that this statement is false. ${ }^{3}$ If it were true, it would imply that if $T$ is a $Z$-representation no two of whose irreducible constituents are $Q$-equivalent, then the irreducible $Z$-constituents of $T$ are unique up to $Z$-equivalence and order of occurrence. We shall give a counterexample to show that this is not the case.

Keep the notation of equations (3)-(5). Let $U$ be the $Z$-representation of $G$ defined by

$$
U(a)=\left(\begin{array}{l|l}
A_{1} & \begin{array}{l}
1 \\
0
\end{array} \\
\hline 1
\end{array}\right), \quad U(b)=\left(\begin{array}{r|r}
B_{1} & 1 \\
-1 \\
\hline 1
\end{array}\right),
$$

so that $U$ has irreducible $Z$-constituents $T_{1}$ and 1 . Set

$$
S=\left(\begin{array}{rrr}
1 & 0 & 1 \\
-1 & -1 & -1 \\
2 & 1 & 1
\end{array}\right), \quad S^{-1}=\left(\begin{array}{rrr}
0 & 1 & 1 \\
-1 & -1 & 0 \\
1 & -1 & -1
\end{array}\right)
$$

Then we find that $S^{-1} U S=V$, where

$$
V(a)=\left(\begin{array}{l|ll}
1 & \frac{1}{0} & 0 \\
A_{2}
\end{array}\right), \quad V(b)=\left(\begin{array}{l|ll}
1 & \frac{0}{1} \\
B_{2}
\end{array}\right) .
$$

Thus, $V$ has irreducible $Z$-constituents $1, T_{2}$. Since $U \sim_{Z} V$, we have our counterexample.

Keeping the notation and hypotheses of (D), the correct version (proved by Diederichsen) is

$\left(\mathrm{D}^{\prime}\right)$ Once the order of occurrence of the $U_{i}$ is fixed, then they are unique up to $Z$-equivalence. In other words, if

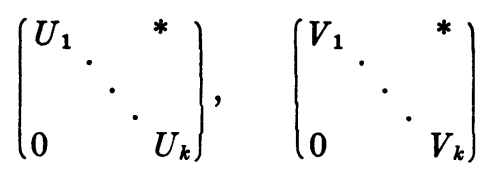

are a pair of $Z$-equivalent $Z$-representations of a finite group such that

$$
U_{i} \sim{ }_{Q} V_{i},
$$$$
1 \leqq i \leqq k,
$$

and such that for $i \neq j$, no irreducible constituent of $U_{i}$ is $Q$-equivalent to any irreducible constituent of $U_{j}$, then in fact $U_{i} \sim_{z} V_{i}, 1 \leqq i \leqq k$.

3 The flaw in Diederichsen's argument is this: he shows that the order of occurrence of the irreducible constituents may be changed at will by unimodular transformation. Unfortunately, he overlooks the fact that such transformations may change the integral classes of the constituents which are involved. 


\section{REFERENCES}

1. F. E. Diederichsen, Über die Ausreduktion ganzzahliger Gruppendarstellungen bei arithmetischer Äquivalenz, Abh. Math. Sem. Hansischen Univ. vol. 13 (1940) pp. 357-412.

2. J.-M. Maranda, On P-adic integral representations of finite groups, Canad. J. Math. vol. 5 (1953) pp. 344-355.

3. H. Zassenhaus, Neuer Beweis der Endlichkeit der Klassenzahl bei unimodularer Äquivalenz endlicher ganzzahliger Substitutionsgruppen, Abh. Math. Sem. Hansischen Univ. vol. 12 (1938) pp. 276-288.

UNIVERSITY OF ILLINOIS

\section{A THEOREM ON FACTORIZABLE GROUPS ${ }^{1}$}

\section{WALTER FEIT}

A group $G$ is said to be factorizable if it contains proper subgroups $H, K$ with the property that $G=H K$. Several results are known which state that $G$ is not simple if $H$ and $K$ satisfy suitable assumptions. The following theorem and its corollaries are of a similar nature but apply only to groups of odd order. The proof is based on a theorem of $\mathrm{H}$. Wielandt (see [2]) which generalized earlier results of W. Burnside and I. Schur.

Theorem. Let $G$ be a group of odd order and let $M$ be a maximal subgroup of $G$. Suppose that $A$ is an abelian subgroup of $G$, which has at least one cyclic Sylow subgroup, such that $G=A M$. Then either $G$ has prime order or $G$ contains a proper normal subgroup $N$ which is contained in either $A$ or in $M$.

Proof. Assume that no proper normal subgroup of $G$ is contained in $M$. Suppose first that $D=A \cap x M x^{-1} \neq\{1\}$, for some element $x$ in $G$. Since $A$ is abelian and since every subgroup of $G$ conjugate to $M$ is of the form $y M y^{-1}$ for some element $y$ in $A$, it follows that $D$ is contained in every subgroup conjugate to $M$. Hence the intersection of all subgroups conjugate to $M$ is a proper normal subgroup of $G$ which is contained in $M$. This contradicts our assumption. Hence $A \cap x M x^{-1}=\{1\}$ for every element $x$ in $G$.

Let $\pi$ be the permutation representation of $G$ induced by the subgroup $M$. As the kernel of $\pi$ is contained in $M$, it follows from the assumptions that $\pi$ is faithful. As $M$ is a maximal subgroup of $G$, $\pi(G)$ is a primitive group of permutations. Since $A$ intersects no

Received by the editors October 12, 1959.

1 This work was supported by the Office of Ordnance Research. 